\title{
Emergencias endocrinas en pediatría: diagnóstico y manejo
}

\author{
Consuelo Aránguiz G a, 0 scar Trujillo la, \\ María Loreto Reyes G. \\ Diagnosis and management of \\ endocrine emergencies in pediatrics
}

The study of endocrine emergencies in childhood is important due to their high mortality and residual morbidity, that can be reduced with an adequate diagnosis and/or therapy. In this article, we review hypoglycemia, adrenal crisis, hypocalcemia, hypercalcemia and thyroid storm in children, with focus on initial diagnostic approach and management (Rev Méd Chile 2005; 133: 1371-80).

(Key Words: Adrenal insufficiency; Hypercalcemia; Hypocalcemia; Hypoglycemia of infancy; Thyroid crisis)

Recibido el 17 de junio, 2004. Aceptado el 15 de abril, 2005.

Departamento de Pediatría, Unidad de Endocrinología, Facultad de Medicina, Pontificia Universidad Católica de Chile.

anterno séptimo año, Escuela de Medicina, Facultad de Medicina, Pontificia Universidad Católica de Chile.

\begin{abstract}
A
pesar de su baja incidencia en el ámbito pediátrico, las emergencias derivadas de patologías del sistema endocrino revisten especial importancia, dado que los síntomas suelen ser poco específicos y el retraso en el diagnóstico y el inicio de la terapéutica adecuada significa un aumento en la morbimortalidad ${ }^{1}$. Revisiones sistemáticas y actualizadas de las emergencias endocrinas en la edad pediátrica son escasas, lo que dificulta el estudio de pediatras y médicos generales que se enfrentan a estos problemas.
\end{abstract}

Correspondencia a: Dra. María Loreto Reyes G. Departamento de Pediatría, Pontificia Universidad Católica de Chile. Lira 85 Piso 5. Santiago, Chile. Fono: 3543402. Fax: 6384307. E mail: mlreyes@med.puc.cl
En el presente artículo se discutirán la aproximación diagnóstica y manejo inicial de las siguientes entidades: hipoglicemia, crisis suprarrenal, hipocalcemia, hipercalcemia y tormenta tiroidea.

Hipoglicemia. La hipoglicemia es uno de los trastornos metabólicos más frecuentes en pediatría. Su diagnóstico y tratamiento precoz son esenciales para le prevención de secuelas neurológicas ${ }^{2}$.

La sintomatología está dada tanto por los efectos directos de la disminución de aporte energético al sistema nervioso central, como por la respuesta adrenérgica. Los síntomas son inespecíficos. En el período neonatal, son letargia, apatía, flacidez, apnea, llanto débil, temblor, irritabilidad, convulsiones, coma. En el niño mayor, la glucopenia cerebral se manifiesta como cefalea, 
visión borrosa, ataxia, irritabilidad, somnolencia, estupor, coma, convulsiones o equivalentes convulsivos, temblores y como secuelas puede ocasionar daño neurológico permanente. La glucopenia muscular se presenta en forma de hipotonía, debilidad, calambres, trastornos del ritmo cardíaco. La estimulación del sistema nervioso simpático da cuenta de sudoración, taquicardia, angustia, náuseas, vómitos y palidez por vasoconstricción ${ }^{2-4}$.

El diagnóstico se confirma con una glicemia menor de $50 \mathrm{mg} / \mathrm{dL}$. Esto es válido tanto para neonatos como para niños de otras edades. Las cintas reactivas son poco confiables, por su escasa sensibilidad en niveles bajos de glicemia 4 .

Las causas de hipoglicemia varían según la edad de presentación y la naturaleza transitoria o permanente de la hipoglicemia. En los neonatos, la mayoría de las causas son transitorias, siendo el resultado de una producción disminuida o utilización aumentada de glucosa (ej: neonato prematuro, neonato hijo de madre diabética, distrés neonatal). La hipoglicemia persistente en el periodo neonatal y lactantes menores es causada por hiperinsulinismo (defectos en canales de potasio o enzimas relacionadas con el metabolismo de células $\beta$, adenoma de células $ß$, síndrome de Beckwith-Wiedemann), hipopituitarismo o enfermedades metabólicas. En lactantes mayores, preescolares y escolares, la hipoglicemia cetócica es la causa más común, se presenta en niños entre 18 meses y 5 años luego de ayuno prolongado (más de $16 \mathrm{~h}$ ) y se resuelve espontáneamente entre los 7 y 8 años. El diagnóstico de esta condición es un diagnóstico de descarte. En la Tabla 1 se muestran las distintas causas de hipoglicemia en pediatría según la edad ${ }^{3-6}$.

Es fundamental al diagnosticar una hipoglicemia, instalar una vía venosa y tomar muestras de sangre y orina previo al tratamiento, para realizar luego el estudio etiológico. Las muestras de sangre deben ser tomadas en tubos heparinizados, extraer el plasma y congelarlo. La muestra de orina puede ser congelada para estudios metabóli$\cos ^{2,3}$

Los elementos a determinar en sangre y orina durante la hipoglicemia están señalados en la Tabla 2.

Los resultados de estos exámenes permiten una orientación diagnóstica de acuerdo al flujograma de la Figura 1.
Inmediatamente después de tomar los exámenes de orientación diagnóstica, debe administrarse glucosa endovenosa. Existen distintos protocolos para este efecto, ninguno respaldado por la evidencia. Proponemos un bolo inicial de solución glucosada al 10\%, 2 a 2,5 mL/kg de peso en 2 a $3 \mathrm{~min}$. Esto debería aumentar la glicemia en 35 $\mathrm{mg} / \mathrm{dL}$, si no sucede, volver a dar un nuevo bolo. Luego de alcanzar niveles seguros de glicemia, se establece una infusión continua con solución glucosada al $10 \%$ a una velocidad de 5 a $8 \mathrm{mg} / \mathrm{kg} /$ min en neonatos y de 3 a $5 \mathrm{mg} / \mathrm{kg} / \mathrm{min}$ en los lactantes y niños mayores. Se debe procurar mantener glicemias por sobre $50 \mathrm{mg} / \mathrm{dL}^{3}$.

Bolos de infusión de glucosa al 50\% o 30\% deben ser evitados pues pueden producir hipoglicemias de rebote importantes y cambios osmóticos rápidos deletéreos ${ }^{3}$.

De no observar respuesta luego de dos bolos de glucosa, debe administrarse un bolo de hidrocortisona $5 \mathrm{mg} / \mathrm{kg}$ intravenoso. Previo a la administración de hidrocortisona se recomienda tomar muestras para ACTH y cortisol ${ }^{3}$.

Frente a la presencia de neonatos macrosómicos, onfalocele, macroglosia o la necesidad de infusión de glucosa a mayor velocidad para mantener glicemias estables alta (cargas >10-15 $\mathrm{mg} / \mathrm{kg} / \mathrm{min}$ ), debe sospecharse hiperinsulinismo. $\mathrm{Si}$ ésto se confirma, se puede utilizar diazoxide 5 a $20 \mathrm{mg} / \mathrm{kg} /$ día, dividido en 2 a 3 dosis. Este medicamento abre el canal de potasio ATP dependiente y aumenta la secreción de epinefrina, aumentando la gluconeogénesis e inhibiendo la secreción de insulina ${ }^{3,5}$.

Una vez estable el paciente, se podrá determinar la etiología de la hipoglicemia e iniciar el tratamiento específico correspondiente.

Crisis suprarrenal. La crisis suprarrenal (CS) corresponde a la presentación aguda de la insuficiencia suprarrenal, con pérdidas de sal, colapso circulatorio e hipoglicemia. Es más frecuente en neonatos y lactantes, pero puede verse a cualquier edad, especialmente asociado a uso crónico de terapia corticoidal ${ }^{7}$. Patologías agudas, cirugías, traumas, estrés emocional, exposición a calor excesivo, uso de diuréticos o ayuno prolongado pueden precipitar una crisis en un niño predispuesto. El eje hipotálamo-hipófisis-suprarrenal (HHS) es fundamental en la respuesta fisiológica 
- em ERGEN CIAS EN D O CRINAS en PEDIATRÍA - C Aránguiz et al

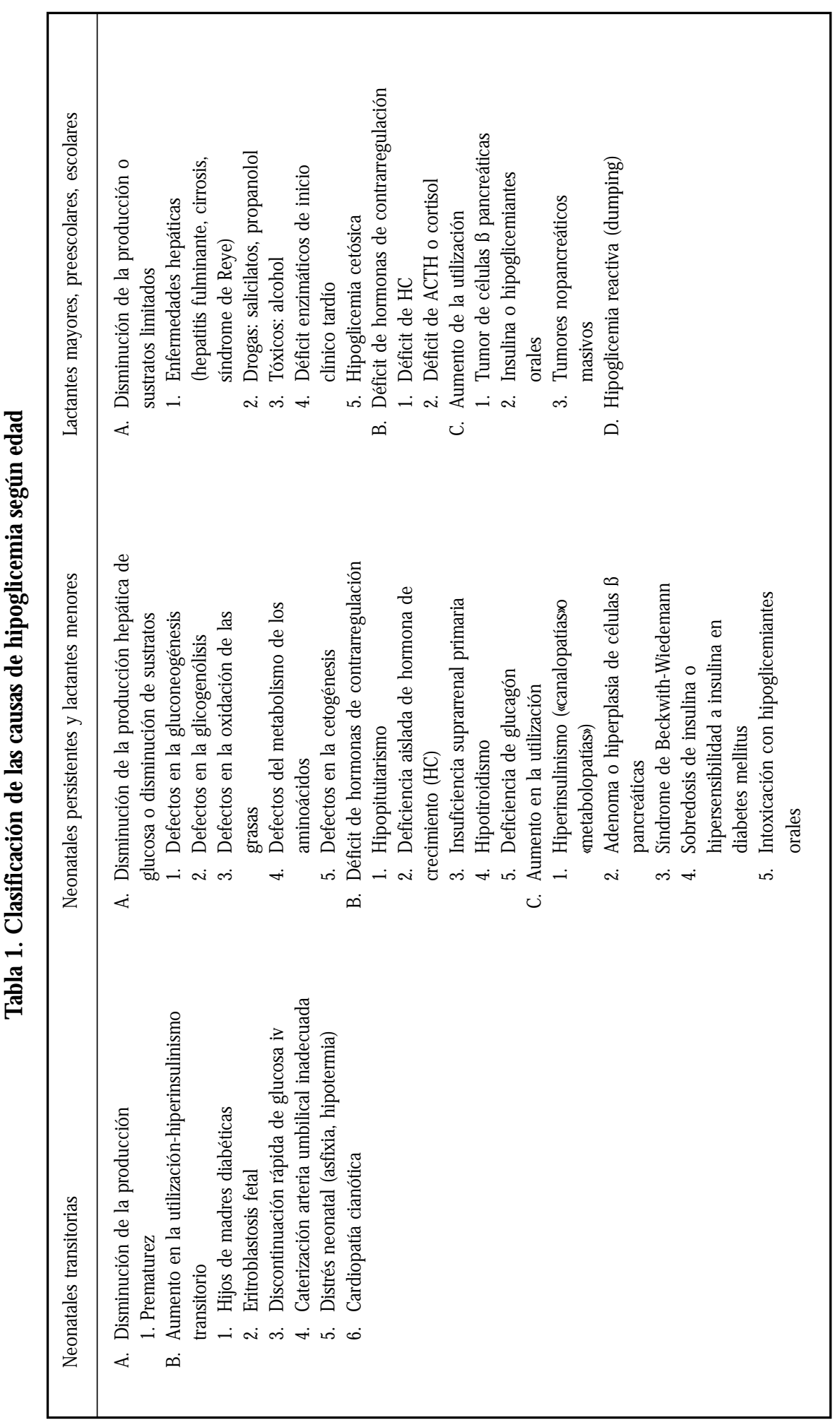


Tabla 2. Exámenes durante hipoglicemia

\begin{tabular}{|ll|}
\hline Sangre venosa & \\
\hline Gases & Insulina \\
Lactato/Piruvato & Péptido c \\
Cuerpos cetónicos & Cortisol \\
Acidos grasos libres & Hormona de crecimiento \\
Amonio & Hormonas tiroideas \\
Carnitina total/libre & ACTH \\
\hline Orina & \\
\hline Cuerpos cetónicos & \\
Sustancias reductoras & \\
Acidos grasos & \\
\hline
\end{tabular}

frente a estímulos estresantes, si las demandas de cortisol no pueden satisfacerse, se desarrolla una $\mathrm{CS}^{7-9}$.

Las causas de insuficiencia suprarrenal pueden ser primarias, si la falla se origina en la glándula suprarrenal, o secundarias, si se debe a una patología hipofisiaria o hipotalámica. A diferencia de los adultos, en pediatría existe una mayor proporción de etiologías congénitas comparadas con las adquiridas ${ }^{7,8}$. En la Tabla 3 se presentan las etiologías más frecuentes. Cualquiera de estos trastornos pueden presentarse como una $\mathrm{CS}^{7}$. Existen ciertas características clínicas que orientan al origen del defecto. En las primarias puede

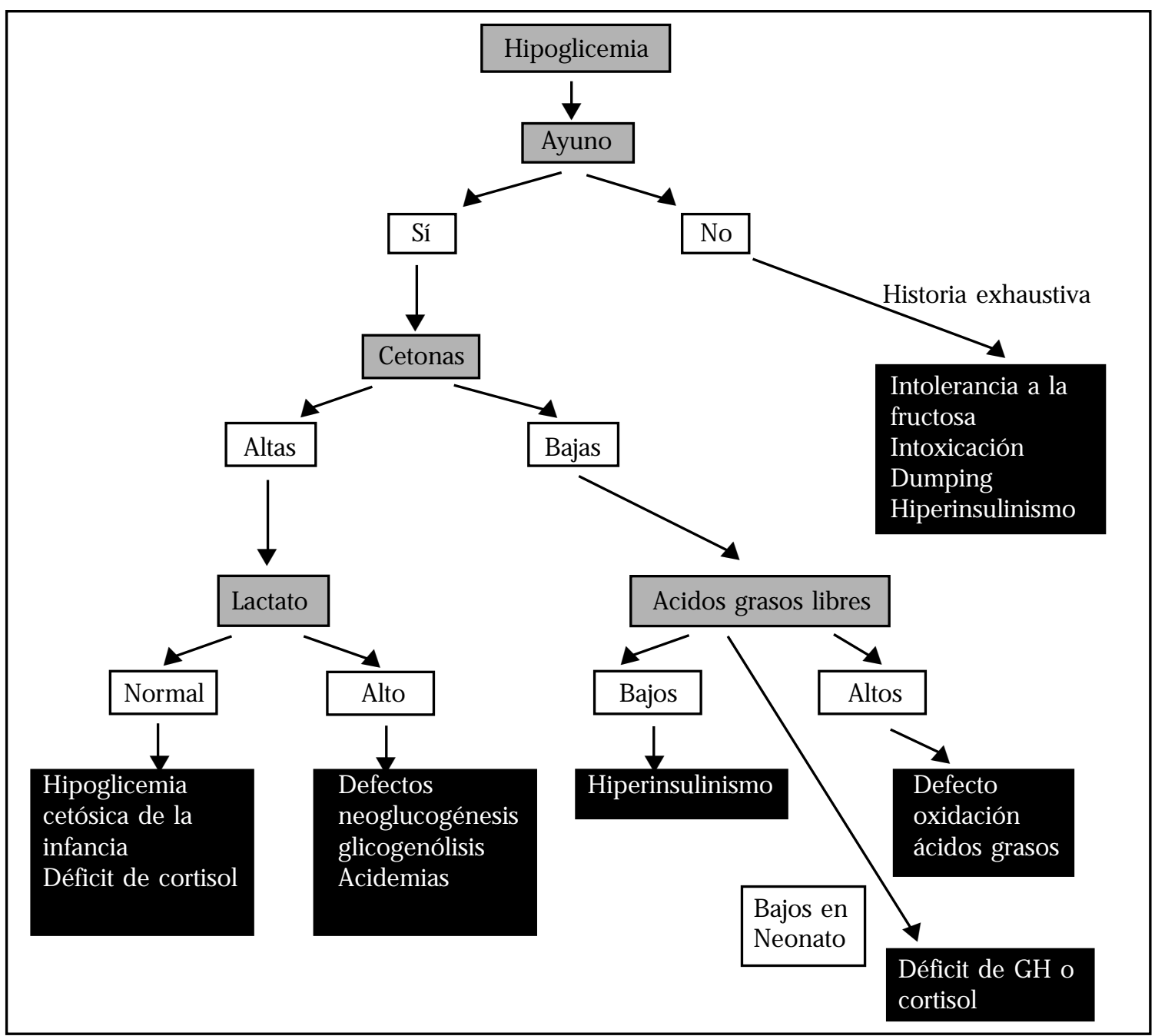

Figura 1. Flujograma de orientación diagnóstica hipoglicemias. GH = Hormona de crecimiento. 
Tabla 3. Etiologías más frecuentes de insuficiencia suprarrenal

\begin{tabular}{|c|c|}
\hline $\begin{array}{l}\text { Causas primarias } \\
\text { Congénitas }\end{array}$ & $\begin{array}{l}\text { Causas secundarias } \\
\text { Congénitas }\end{array}$ \\
\hline $\begin{array}{l}\text { Hiperplasia suprarrenal congénita } \\
\text { Hipoplasia suprarrenal congénita } \\
\text { Insensibilidad a ACTH } \\
\text { Hipoaldosteronismo } \\
\text { Pseudohipoaldosteronismo } \\
\text { Adrenoleucodistrofia }\end{array}$ & $\begin{array}{l}\text { Panhipopituitarismo aislado } \\
\text { Asociado a defectos anatómicos } \\
\text { Idiopático }\end{array}$ \\
\hline Adquiridas & Adquiridas \\
\hline $\begin{array}{l}\text { Autoinmune } \\
\text { Infecciones: } \\
\text { - } \quad \text { Tuberculosis, sífilis } \\
\text { - } \text { VIH } \\
\text { - } \quad \text { Meningococo (Waterhouse-Friderichsen) } \\
\text { Iatrogénica (antibiótico, antimicóticos) } \\
\text { Trombosis vena suprarrenal } \\
\text { Hemorragias suprarrenales }\end{array}$ & $\begin{array}{l}\text { Uso de esteroides crónico } \\
\text { Tumores } \\
\text { Autoinmune }\end{array}$ \\
\hline
\end{tabular}

existir hiperpigmentación, dada por el aumento concomitante de la secreción de hormona estimulante de melanocitos, hiperkalemia y mayor hipotensión. En las CS por falla secundaria no hay hiperkalemia, ya que la regulación de la secreción de aldosterona es independiente del HHS. Además, pueden encontrarse otros signos o síntomas de hipopituitarismo $0^{8,9}$.

La CS suele presentarse de forma abrupta, aunque puede haber fatiga, irritabilidad, náuseas y dolor abdominal precediendo el episodio. El niño presenta vómitos, deshidratación e irritabilidad, que pueden ser seguidos por hipotermia, confusión, coma y falla circulatoria. En el laboratorio básico, se puede pesquisar hiponatremia, hiperkalemia, leve acidosis metabólica, hipoglicemia y eosinofilia o linfocitosis ${ }^{8,9}$.

Frente a la sospecha clínica de una CS, se debe optar por una actitud agresiva de manejo, dada la alta mortalidad asociada a un retraso en el tratamiento. Antes, deben tomarse muestras de sangre para medir concentración plasmática de cortisol y ACTH y de esta forma confirmar el diagnóstico y orientar la etiología. En caso de no poder procesar de inmediato la muestra, refrigerar.

Como tratamiento se recomienda:

- Reposición de volumen: iniciar bolos de $10 \mathrm{~mL}$ $\mathrm{kg}$ de solución de $\mathrm{NaCI}$ 0,9\% y mantener infusión de acuerdo al estado hemodinámico del paciente. En general los requerimientos de volumen durante las primeras $24 \mathrm{~h}$ son de 100$120 \mathrm{~mL} / \mathrm{kg}$, cuando el peso del niño es menor a $20 \mathrm{~kg}$ y de $75 \mathrm{~mL} / \mathrm{kg}$ cuando pesa más de 20 $\mathrm{kg}$. Se debe infundir $20-25 \%$ de este volumen en las primeras dos horas.

- Hipoglicemia: adicionar infusión de solución glucosada al 5\% ó $10 \%$ de acuerdo al nivel de glicemia.

- Corticoides sistémicos: usar hidrocortisona como droga de elección, ya que es el esteroide de uso intravenoso que tiene mayor efecto mineralocorticoide. Se debe partir con bolos intravenosos de $50 \mathrm{mg} / \mathrm{m}^{2}$ de superficie corporal (estimación rápida: lactantes $25 \mathrm{mg}$, niños mayores $50 \mathrm{mg}$ ). En caso de no tener una vía accesible por el grado de colapso circulatorio, 
se puede administrar de forma intramuscular doblando la dosis. Continuar con dosis entre 80 y $100 \mathrm{mg} / \mathrm{m}^{2} /$ día dividido en cuatro dosis.

- $\quad \alpha$ fluoridrocortisona 0,05-0,15 mg/día oral si se sospecha déficit de mineralocorticoides ${ }^{8}$.

Se debe intentar identificar tanto la causa subyacente como la causa desencadenante para su manejo específico.

Hipocalcemia. El calcio en el humano se encuentra en $99 \%$ en el esqueleto y sólo $1 \%$ en el plasma. Su nivel normal varía entre 8,5 y $10,5 \mathrm{mg} / \mathrm{dL}^{10}$ y está regulado, fundamentalmente, por parathormona (PTH), vitamina D y calcitonina. La PTH eleva los niveles de este mineral a través de la estimulación de la resonción ósea, de la reabsorción tubular renal y de la activación de la vitamina D. La vitamina D, por su parte, aumenta la absorción intestinal de calcio y la resorción ósea. La calcitonina es la única que disminuye el calcio sanguíneo, inhibiendo la actividad osteoclástica y aumentando su excreción urinaria1,11.

Se define como hipocalcemia los valores de calcio sérico total menores a $8,5 \mathrm{mg} / \mathrm{dL}$ e iónico menor de $4 \mathrm{mg} / \mathrm{dL}$ La sintomatología clínica suele aparecer con valores menores a $6 \mathrm{mg} / \mathrm{dL}^{10}$.

En pediatría, diversas patologías conducen a este estado , entre las cuales destaca el hipoparatiroidismo, causa más frecuente de hipocalcemia de curso crónico. En la Tabla 4 se detallan las etiologías más comunes ${ }^{10-13}$.

Tabla 4. Patologías causantes de hipocalcemia en pediatría

Hipoparatiroidismo

Congénito

Adquirido

Pseudohipoparatiroidismo

Alteraciones de Vitamina D
Neonatal transitorio

Hereditario

Síndrome de Di George

Síndrome de Kenny-Caffey

Post quirúrgico

Hemosiderosis

Enfermedad de Wilson

Síndrome poliglandular, Tipo I

Resistencia a PTH

Baja exposición solar-Baja ingesta

Síndrome de mala absorción

Insuficiencia renal crónica

Raquitismo hipocalcémico tipo I

(deficiencia $1 \alpha$ hidroxilasa)

Raquitismo resistente (resistencia a vitamina D)

Otros 
Las manifestaciones clínicas de la hipocalcemia dependen de la severidad y de la cronicidad. Temblores, parestesia, calambres, debilidad, fatiga, letargia, estatus mental alterado, convulsiones o equivalentes, tetania, papiledema así como estridor, apnea y laringoespasmo se han descrito en hipocalcemias agudas. Las hipocalcemias de larga evolución suelen tener retardo del desarrollo psicomotor y cataratas lenticulares. En el examen físico destacan el movimiento del labio superior al percutir el nervio facial (signo de Chvostek, presente en $10 \%$ de la población sana) y el espasmo carpopedal al ocluir por $3 \mathrm{~min}$ la circulación de una extremidad superior con un manguito de presión (signo de Trousseau, más específico). En el electrocardiograma existe prolongación del intervalo $\mathrm{QT}^{10,11,13}$.

El diagnóstico se confirma con niveles bajo el límite normal para la edad de calcio plasmático (total e ionizado). Se debe tener en cuenta descartar una patología que curse con hipoalbuminemia o alteraciones ácido-base. Se debe entonces cuantificar albúmina y tomar gases venosos. El calcio sérico corregido se obtiene de acuerdo a la siguiente fórmula:

Calcio plasmático corregido $=$

Calcio sérico + 0,8 $(4,0$ - albúmina g/dL)

En caso de alteración ácido-base, se recomienda medir calcio iónico ${ }^{10}$.

Se debe considerar dentro del estudio, niveles de fosfato, PTH, fosfatasas alcalinas, calcio urinario, magnesio, proteínas totales y gases venosos.

La elevación de fosfatos sugiere hipoparatiroidismo. Una PTH baja o inapropiadamente normal durante hipocalcemia, indica hipoparatiroidismo primario, en cambio si la PTH es alta sugiere un pseudohipoparatiroidismo o déficit de vitamina $D$. La elevación de fosfatasas alcalinas requiere descartar un raquitismo hipocalcémico. La hipercalciuria sugiere hipoparatiroidismo familiar hereditario por mutación del sensor de calcio. En relación a la utilidad de la medición de magnesio, es importante destacar que la hipocalcemia responde mal a tratamiento, mientras no se corrige la hipomagnesemia ${ }^{10,11,13}$.

Pacientes sintomáticos severos (laringoespasmos, tetania, convulsiones) constituyen una emergencia endocrina y requieren inmediata suplementación intravenosa. Se debe administrar gluconato de calcio diluido al $5 \%(1 \mathrm{~mL}=4,65 \mathrm{~g}$ de calcio elemental) en dosis de $2 \mathrm{~mL} / \mathrm{kg}$ en infusión lenta con monitoreo cardíaco. Esto puede repetirse hasta desaparición de la sintomatología. Controlar la calcemia una hora después. Luego del manejo de la sintomatología aguda, agregar gluconato de calcio al 10\%, 5-10 mL/kg en $24 \mathrm{~h}$. Controlar la calcemia cada $4 \mathrm{~h}$. Se debe reducir progresivamente el aporte de calcio enintravenoso dentro de las $24 \mathrm{~h}$ posteriores a haber normalizado la calcemia ${ }^{13}$.

$\mathrm{Si}$ las manifestaciones clínicas son menores, puede iniciarse tratamiento oral con carbonato, gluconato o lactato de calcio, $50-100 \mathrm{mg} / \mathrm{kg} /$ día de calcio elemental. En algunas etiologías, además del aporte de calcio oral, es necesario indicar calcitriol 0,20-0,25 $\mu \mathrm{g}$ en recién nacidos y 25-50 $\mathrm{ng} / \mathrm{kg} /$ día dividido en dos dosis en mayores de un mes de vida ${ }^{13}$.

$\mathrm{Si}$ se constata hipomagnesemia, se debe corregir con sulfato de magnesio $25-50 \mathrm{mg} / \mathrm{kg} / \mathrm{dosis}$ cada 4 a $6 \mathrm{~h}$, con dosis única máxima de $2 \mathrm{~g}^{13}$.

Una vez estabilizada la condición clínica debe buscarse y tratarse la patología de base.

Hipercalcemia. En pediatría la hipercalcemia aguda es una entidad poco frecuente y su etiología se encuentra expuesta en la Tabla $5^{11,13,14}$. A diferencia de los adultos, las hipercalcemias asociadas a patología neoplásica son raras y se observan principalmente en leucemias linfoblásticas ${ }^{15}$.

Los síntomas asociados a hipercalcemia aguda suelen presentarse con niveles por sobre $12 \mathrm{mg} /$ $\mathrm{dL}$ y ser severos con niveles mayores a $15 \mathrm{mg} /$ $\mathrm{dL}^{1,11}$. Éstos se expresan a nivel del sistema gastrointestinal, renal y nervioso y son inespecíficos. La clínica sugerente incluye: debilidad, irritabilidad, letargia, confusión, alucinaciones, marcha inestable, anorexia, náusea, vómitos, constipación, polidipsia y poliuria (por diabetes insípida nefrogénica), que pueden llevar a la deshidratación. En el examen físico pueden hallarse bradicardia, sensorio deprimido, hiporreflexia y debilidad muscular proximal. En el electrocardiograma destaca una disminución del segmento ST que da cuenta de un QT menor ${ }^{11,14}$.

El diagnóstico se confirma con la medición de calcio plasmático, teniendo en cuenta, al igual que para hipocalcemia, los niveles de albúmina y estado ácido-base. 
Tabla 5. Principales causas de hipercalcemia
Hiperparatiroidismo
Familiar o aislado
Hipervitaminosis D
Neoplásico
Enfermedades granulomatosas
Leucemias linfoblásticas, linfomas
Uso de diuréticos tiazídicos
Síndromes genéticos
Sarcoidosis, tuberculosis
Otros
Síndrome de Williams, condrodisplasia metafisiaria de Jansen
Inmovilización prolongada, síndrome de álcali-
leche, Acidosis tubular renal

Dentro del estudio etiológico inicial, considerar cuantificar fosfemia, fosfatasas alcalinas, creatininemia, PTH y $25 \mathrm{OH}$ vitamina D.

$\mathrm{Si}$ el calcio plasmático es mayor a $15 \mathrm{mg} / \mathrm{dL} 0$ mayor a $13 \mathrm{mg} / \mathrm{dL}$ asociado a síntomas, debe iniciarse una agresiva terapia cuyos objetivos son optimizar la excreción de calcio, disminuir su salida desde el compartimiento óseo y eventualmente, aumentar los depósitos en éste ${ }^{11,13}$.

El cuidado médico debe incluir monitorización cardiorrespiratoria permanente, dado el riesgo potencial de ectopías ventriculares.

Debe administrarse volumen con solución fisiológico (3.000-4.000 mL/m²/día), tanto para reponer las pérdidas dadas por la patología, como para mejorar la excreción urinaria de calcio. La adición de un diurético de asa (furosemida $1 \mathrm{mg} /$ kg cada $6 \mathrm{~h}$ ), optimiza este proceso ${ }^{11,13}$.

El uso de corticoides, como hidrocortisona (5 $\mathrm{mg} / \mathrm{kg} /$ dosis cada $6 \mathrm{~h}$ ), tiene poco efecto sobre la resorción ósea, pero disminuye la absorción intestinal y aumenta la excreción urinaria. Su mayor eficacia se logra en neoplasias, enfermedades granulomatosas y toxicidad por vitamina $\mathrm{D}^{11}$.

Los bifosfonatos actúan bloqueando la resorción ósea. Su uso en pediatría ha sido limitado, pero existe cierta experiencia efectiva con pamidronato $(0,5-1 \mathrm{mg} / \mathrm{kg}$ en infusión endovenosa durante 4 horas). Los efectos adversos reportados son fiebre baja, mialgias, linfopenia, gastritis, dolor óseo e hipocalcemia transitoria ${ }^{16}$.

Otro medicamento coadyuvante es la calcitonina (4-8 U/ kg cada $6 \mathrm{~h}$ endovenoso), que debería considerarse en hipercalcemias refractarias ${ }^{11}$.
$\mathrm{Si}$ a pesar de todas estas medidas el paciente se mantiene con niveles altos de calcio, está indicada la hemodiálisis.

Tormenta tiroidea. Se denomina así a un estado grave de hipertiroidismo, asociado a riesgo vital. Ocurre siempre en el contexto de un hipertiroidismo no controlado. Infecciones, trauma, cirugía, cetoacidosis diabética o aporte excesivo de yodo son algunos desencadenantes de las crisis. Dada la baja frecuencia de esta condición, no existen estudios que clarifiquen la fisiopatología, ya que los perfiles de niveles sanguíneos de triyodotironina (T3) y tiroxina (T4) pueden ser idénticos a los de pacientes con hipertiroidismo no complicado. Los mecanismos que podrían explicar por qué estos factores pueden agravar el hipertiroidismo, se relacionan con la liberación de citoquinas y alteración aguda inmunológica ${ }^{17}$.

En pediatría, la patología hipertiroidea es poco frecuente, por lo que es raro ver tormentas tiroideas. La enfermedad de Graves y las tiroiditis autoinmunes son las causas principales de tormenta tiroidea ${ }^{17,18}$.

Los elementos que permiten plantear el diagnóstico de tormenta tiroidea son:

- Paciente con antecedentes de hipertiroidismo preexistente (bocio, exoftalmos, síntomas de tirotoxicosis previos).

- Fiebre siempre presente. Puede llegar a hipertermia $\left(40^{\circ} \mathrm{C}\right)$ y acompañarse de deshidratación.

- Trastornos de área psíquica: agitación, delirio, hiperkinesia, psicosis, apatía, estupor, coma. 
- Sudoración profusa.

- Náuseas, vómitos, dolor abdominal al inicio de la crisis.

- Taquicardia extrema (140 x min), arritmias, insuficiencia cardíaca congestiva, edema pulmonar ${ }^{17,19}$.

El estudio inicial debe incluir medición de hormonas tiroideas (T3, T4, T4 libre, TSH), electrolitos plasmáticos y electrocardiograma. No se debe esperar los resultados de tales exámenes para iniciar tratamiento, pues un retraso puede ser fatal.

El objetivo terapéutico es disminuir rápidamente las hormonas tiroideas, controlar la causa precipitante, la temperatura corporal y las complicaciones hidroelectrolíticas, cardíacas (insuficiencia cardíaca, arritmias) y metabólicas (hipoglicemia). El paciente debe ser hospitalizado en una unidad de cuidados intensivos ${ }^{17}$.

Para disminuir las hormonas tiroideas se utilizan drogas antitimideas, y yodo en altas dosis para bloquear la liberación de T3 y T4 desde la glándula.

El propiltiuracilo (PTU) es la droga de elección, ya que actúa tanto en la inhibición de síntesis de hormonas tiroideas como en la conversión periférica de T4 a T3. La dosis es de $10 \mathrm{mg} / \mathrm{kg} /$ día cada 8 $\mathrm{h}$, por vía oral. En pacientes inconscientes se

\section{REFERENCIAS}

1. Bagdade J. Endocrine emergencies. Med Clin North Am 1986; 70: 1111-28.

2. Lee PJ, Leonard JV. Hypoglycaemia. En: Clinical paediatric endocrinology. 3a Edición. Cambridge: Editorial Blackwell Sciences 1995; 677-693.

3. LafRANCHI S. Hypoglycemia of infancy and childhood. Pediatr Clin North Am 1987; 34: 961-82.

4. Merico MV. Hipoglicemia. En: Beas F. ed. Endocrinología del niño y el adolescente. 1a Edición. Santiago: Editorial Mediterráneo, 1997; 258-74.

5. Cosgrove K, Shepherd R, Fernández E. Genetics and pathophysiology of hyperinsulinism in infancy. Horm Res 2004; 61: 270-88.

6. Saudubray JM, Lonlay P, Touati G et al. Genetic hypoglycaemia in infancy and childhood: pathophyiology and diagnosis. J Inherit Metab Dis FALTA AÑO 23: 197-214. requerirá aporte por sonda nasogástrica o vía rectal, ya que no existe formulación parenteral ${ }^{17,20}$.

El lopodato de sodio o solución de Lugol disminuye de forma aguda la liberación de hormona tiroidea e inhibe la conversión periférica de T4 a T3. Debe indicarse 4 h después de iniciada la administración de PTU, pues éste inhibe la síntesis de hormona tiroidea estimulada por el yodo evitando un aumento de las reservas de yodo en la glándula y mayor producción de hormonas ${ }^{17}$.

Se indica también corticoides pues inhiben la conversión periférica, además de actuar sobre la fiebre y mantener la normotensión ${ }^{17,19}$.

No existen recomendaciones basadas en evidencia para la dosificación de dexametasona ni lugol en pediatría, pero se sugieren dosis de dexametasona de $0,15 \mathrm{mg} / \mathrm{kg} /$ día dividido cada $6 \mathrm{~h}$ y de lugol de $1 \mathrm{gota} / \mathrm{kg} /$ día dividido cada $8 \mathrm{~h}$.

Para el manejo sintomático (taquicardia, arritmias, temblor, sudoración) se utilizan beta bloqueadores (propanolol 0,2 a 0,5 mg/kg/día), teniendo especial cuidado con la aparición de signos de insuficiencia cardíaca ${ }^{17-19}$.

La pirexia debe manejarse con medidas físicas o con acetamifeno. El uso de aspirina está proscrito, ya que desplaza T4 de la globulina ligante de tiroxina (TBG).

7. Forest MG. Adrenal steroid deficiency states. En: Clinical paediatric endocrinology. 3a Edición. Cambridge: Editorial Blackwell Sciences 1995; 453-61.

8. OelKers W. Adrenal insufficiency. N Engl J Med 1996; 335: 1206-12.

9. BuRgueÑo M. Insuficiencia suprarrenal aguda. En: Beas F. ed. Manual de endocrinología pediátrica. 1a Edición. Santiago: Editorial Mediterráneo, 1993; 181-95.

10. SHANE E. Hypocalcemia: pathogenesis, differential diagnosis, and management. En: Primer on the metabolic bone diseases and disorders of mineral metabolism. 4a Edición. Filadelfia: Editorial Lippincot Williams \& Wilkins,1999; 223-5.

11. Bringhurst F, Demay M, Kronenberg H. Hormones and disorders of mineral metabolism. En: Wilson $\mathrm{J}$. ed. Williams textbook of endocrinology, $10^{\text {a }}$ Edición. Philadelphia: Editorial W. B. Saunders, 2003; 1323-48. 
12. Fanella G, Elahna P, Dimartino-Nardi J et al. Transient congenital hypoparathyroidism: resolution and recurrence in chromosome 22q11 deletion. J Pediatr 1996; 128: 563-7.

13. KRUSE K. Disorders of calcium and bone metabolism. En: Clinical paediatric endocrinology. $3^{\mathbf{a}}$ Edition. Cambridge: Editorial Blackwell Sciences 1995; 736-60.

14. Langman C. Hypercalcemic syndromes in infants and children. En: Primer on the metabolic bone diseases and disorders of mineral metabolism. $4^{\text {a }}$ Edición. Filadelfia: Editorial Lippincot Williams \& Wilkins, 1999; 219-22.

15. Mckay C, Furman W. Hypercalcemia complicating childhood malignancies. Cancer 1993; 72: 256-60.
16. Lteif A, Zimmerman D. Bisphosphonates for treatment of childhood hypercalcemia. Pediatrics 1998; 102: 990-3.

17. Larsen P, Davies T, Hay I. The thyroid gland. En: Wilson J. ed. Williams textbook of endocrinology, $10^{a}$ Edición. Philadelphia: Editorial W. B. Saunders, 2003; 413-14.

18. KRaIEM Z, NewField RS. Grave's disease in childhood. J Pediatr End Metab 2001; 14: 229-43.

19. Cubilos L, Espinoza R. Tormenta timoidea. En: Cubillos L, ed. Manual de urgencias médicas. 1a Edición. Santiago: Editorial Mediterráneo, 1992; 158-9.

20. IorKansKy S, Herzovich V. Hipertiroidismo infantojuvenil. En: Beas F. ed. Endocrinología del niño y el adolescente. 1a Edición. Santiago: Editorial Mediterráneo, 1997; 160-71.

\section{Agradecimientos}

Agradecemos a los doctores Eugenio Arteaga y Gloria Durán por la revisión de algunas partes de este manuscrito. 